Article

\title{
Involvement of Lactate and Pyruvate in the Anti-Inflammatory Effects Exerted by Voluntary Activation of the Sympathetic Nervous System
}

\author{
Jelle Zwaag ${ }^{1,2,+}$, Rob ter Horst ${ }^{2,3,+}{ }^{-}$, Ivana Blaženović ${ }^{4,5}$, Daniel Stoessel ${ }^{5,6,7}$, \\ Jacqueline Ratter ${ }^{2,3}$, Josephine M. Worseck ${ }^{5}$, Nicolas Schauer ${ }^{5}$, Rinke Stienstra ${ }^{2,3,8}$, \\ Mihai G. Netea ${ }^{2,3,9}$, Dieter Jahn ${ }^{4}$, Peter Pickkers ${ }^{1,2}$ and Matthijs Kox ${ }^{1,2, *(1)}$ \\ 1 Department of Intensive Care Medicine, Radboud Institute for Molecular Life Sciences, Radboud University \\ Medical Center, Internal Mail 710 Geert Grooteplein 10, 6500HB Nijmegen, The Netherlands; \\ Jelle.zwaag@radboudumc.nl (J.Z.); peter.pickkers@radboudumc.nl (P.P.) \\ 2 Radboud Center for Infectious Diseases (RCI), Radboud University Medical Center, Internal Mail 710 Geert, \\ Grooteplein 10, 6500HB Nijmegen, The Netherlands; RterHorst@cemm.oeaw.ac.at (R.t.H.); \\ Jacqueline.Ratter@radboudumc.nl (J.R.); Rinke.stienstra@radboudumc.nl (R.S.); \\ Mihai.netea@radboudumc.nl (M.G.N.) \\ 3 Department of Internal Medicine, Radboud Institute for Molecular Life Sciences, Radboud University \\ Medical Center, Internal Mail 710 Geert Grooteplein 10, 6500HB Nijmegen, The Netherlands \\ 4 Institute of Microbiology, Technische Universität Braunschweig, Universitätsplatz 2, \\ 38106 Braunschweig, Germany; ivana.blazenovic@gmail.com (I.B.); d.jahn@tu-bs.de (D.J.) \\ 5 Metabolomic Discoveries, GmbH, Am Mühlenberg 11, 14476 Potsdam, Germany; \\ danielstoessel@yahoo.com (D.S.); josephine@worseck.de (J.M.W.); nschauer@gmail.com (N.S.) \\ 6 Department of Biochemistry and Biology, Universität Potsdam, Am Neuen Palais 10, \\ 14469 Potsdam, Germany \\ 7 Max Planck Institute für Molekulare Pflanzenphysiologie, Am Mühlenberg 1, 14476 Potsdam, Germany \\ 8 Nutrition, Metabolism and Genomics Group, Division of Human Nutrition, Wageningen University, \\ Droevendaalsesteeg 4, 6708 PB Wageningen, The Netherlands \\ 9 Immunology and Metabolism, LIMES Institute, University of Bonn, Carl-Troll-Straße 31, \\ 53115 Bonn, Germany \\ * Correspondence: Matthijs.kox@radboudumc.nl; Tel.: +31243653881 \\ + These authors contributed equally to this work.
}

Received: 20 February 2020; Accepted: 7 April 2020; Published: 10 April 2020

\begin{abstract}
We recently demonstrated that the sympathetic nervous system can be voluntarily activated following a training program consisting of cold exposure, breathing exercises, and meditation. This resulted in profound attenuation of the systemic inflammatory response elicited by lipopolysaccharide (LPS) administration. Herein, we assessed whether this training program affects the plasma metabolome and if these changes are linked to the immunomodulatory effects observed. A total of 224 metabolites were identified in plasma obtained from 24 healthy male volunteers at six timepoints, of which 98 were significantly altered following LPS administration. Effects of the training program were most prominent shortly after initiation of the acquired breathing exercises but prior to LPS administration, and point towards increased activation of the Cori cycle. Elevated concentrations of lactate and pyruvate in trained individuals correlated with enhanced levels of anti-inflammatory interleukin (IL)-10. In vitro validation experiments revealed that co-incubation with lactate and pyruvate enhances IL-10 production and attenuates the release of pro-inflammatory IL- $1 \beta$ and IL- 6 by LPS-stimulated leukocytes. Our results demonstrate that practicing the breathing exercises acquired during the training program results in increased activity of the Cori cycle. Furthermore, this work uncovers an important role of lactate and pyruvate in the anti-inflammatory phenotype observed in trained subjects.
\end{abstract}


Keywords: metabolomics; LPS; endotoxin; pyruvate; lactate; cytokines; inflammation; human endotoxemia; cori cycle; warburg effect

\section{Introduction}

Recent work from our group revealed that the sympathetic nervous system can be voluntarily activated through a training program consisting of exposure to cold, breathing exercises, and meditation [1]. Compared to an untrained control group who did not practice any exercise, a strong increase in endogenous epinephrine levels was observed in trained healthy volunteers shortly after initiation of the learned breathing exercises. This resulted in augmentation of plasma concentrations of interleukin (IL)-10, a pivotal anti-inflammatory cytokine, and suppression of the pro-inflammatory response during experimental human endotoxemia, a standardized and reproducible model of systemic inflammation elicited by lipopolysaccharide (LPS) administration [1]. Activation of the sympathetic nervous system is also known to profoundly impact cellular metabolism [2]. Furthermore, interaction between immune responses and host metabolism, also termed "immunometabolism", has gained renewed interest, as it was recently discovered that metabolic reprogramming of innate immune cells plays a critical role in mounting effective immune responses and in generation of innate immune memory [3,4].

Metabolomic profiling, for instance using reversed phase chromatography coupled to electrospray ionization mass spectrometry (ESI-MS), enables the detection of highly polar compounds in blood [5]. These techniques allow for simultaneous investigation of a large number of metabolites, thereby providing an indication of the "metabolic status" of an individual [6]. Using such an established platform [7], we performed metabolomic profiling of plasma samples obtained in the study described above [1]. Our primary aim was to determine changes in the plasma metabolome of trained subjects [1], and to assess if these changes could play a role in the immunomodulatory effects observed.

\section{Results}

Characteristics of the study population are listed in Table S1 in the Supplementary Material and reveal no differences between the control group and the trained group.

\subsection{Effects of LPS Administration on Plasma Metabolites}

We first examined LPS-induced changes on the plasma metabolome. To this end, we restricted the analyses to the control group. Expectedly, LPS administration resulted in typical flu-like symptoms, fever, hemodynamic changes, and increases in plasma concentration of pro- and anti-inflammatory cytokines (results reported in detail elsewhere [1], baseline, peak, and area under curve (AUC) plasma cytokine levels are provided in Table S2 in the Supplementary Material). We compared levels of plasma metabolites $0,1,2,4$, and $8 \mathrm{~h}$ after LPS administration to those at baseline (one hour before LPS administration). PCA plots are depicted in Figure $1 \mathrm{a}$ and show the clearest separation at $4 \mathrm{~h}$ post-LPS. 

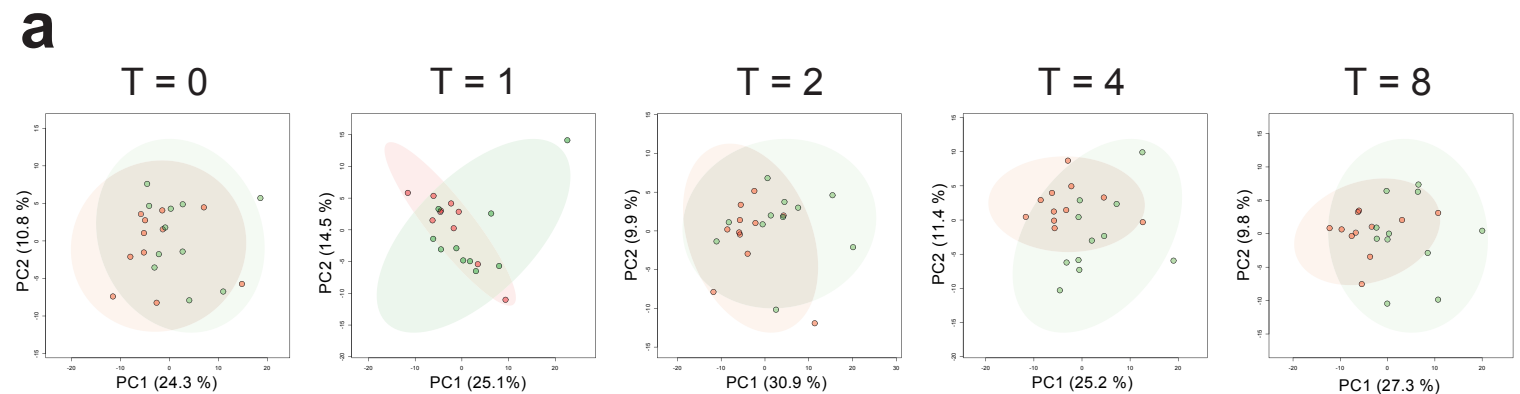

b

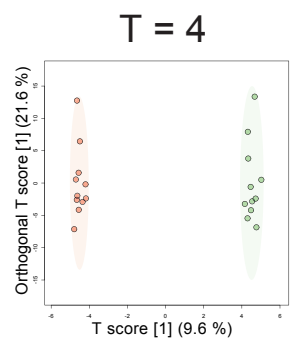

Figure 1. Effects of endotoxemia on the plasma metabolome. (a) Principal component analysis (PCA) of the plasma metabolome at $\mathrm{T}=0$ (just prior to lipopolysaccharide (LPS) administration), 1, 2, 4, and 8 $\mathrm{h}$ following LPS administration in the control group. The red dots indicate baseline (one hour before LPS administration) and the green dots are the timepoints, indicated above the graphs. The shaded areas indicate the $95 \%$ confidence intervals. (b) Orthogonal partial least squares-discriminant analysis (OPLS-DA) of the plasma metabolome at $4 \mathrm{~h}$ after LPS administration in the control group. The red dots indicate baseline (one hour before LPS administration) and the green dots indicate $4 \mathrm{~h}$ post-LPS administration. The shaded areas indicate the $95 \%$ confidence intervals.

Subsequently, we constructed orthogonal partial least squares-discriminant analysis (OPLS-DA) models. Permutation tests revealed that only the model constructed for the comparison of $\mathrm{T}=4 \mathrm{~h}$ versus baseline showed significant separation $\left(1+3\right.$ components, $R^{2} X_{\text {cum }}: 0.448, R^{2} Y_{\text {cum }}: 0.997, Q^{2}$ cum: 0.404; permutation tests: $\mathrm{R}^{2} \mathrm{Y}: p<0.001, \mathrm{Q}^{2}: p=0.03$, score plot depicted in Figure $1 \mathrm{~b}$ ). Differential analyses revealed 98 significantly altered metabolites out of a total of 224 identified metabolites (false discovery rate $[\mathrm{FDR}]$-adjusted $p$-value of $<0.1$ ) across all timepoints. The top 25 significantly altered metabolites are depicted in Figure 2 (all decreased compared with baseline). A complete overview of all metabolites on all timepoints is provided in Tables S3 and S4 in the Supplementary Material. 
Control group (compared to baseline)

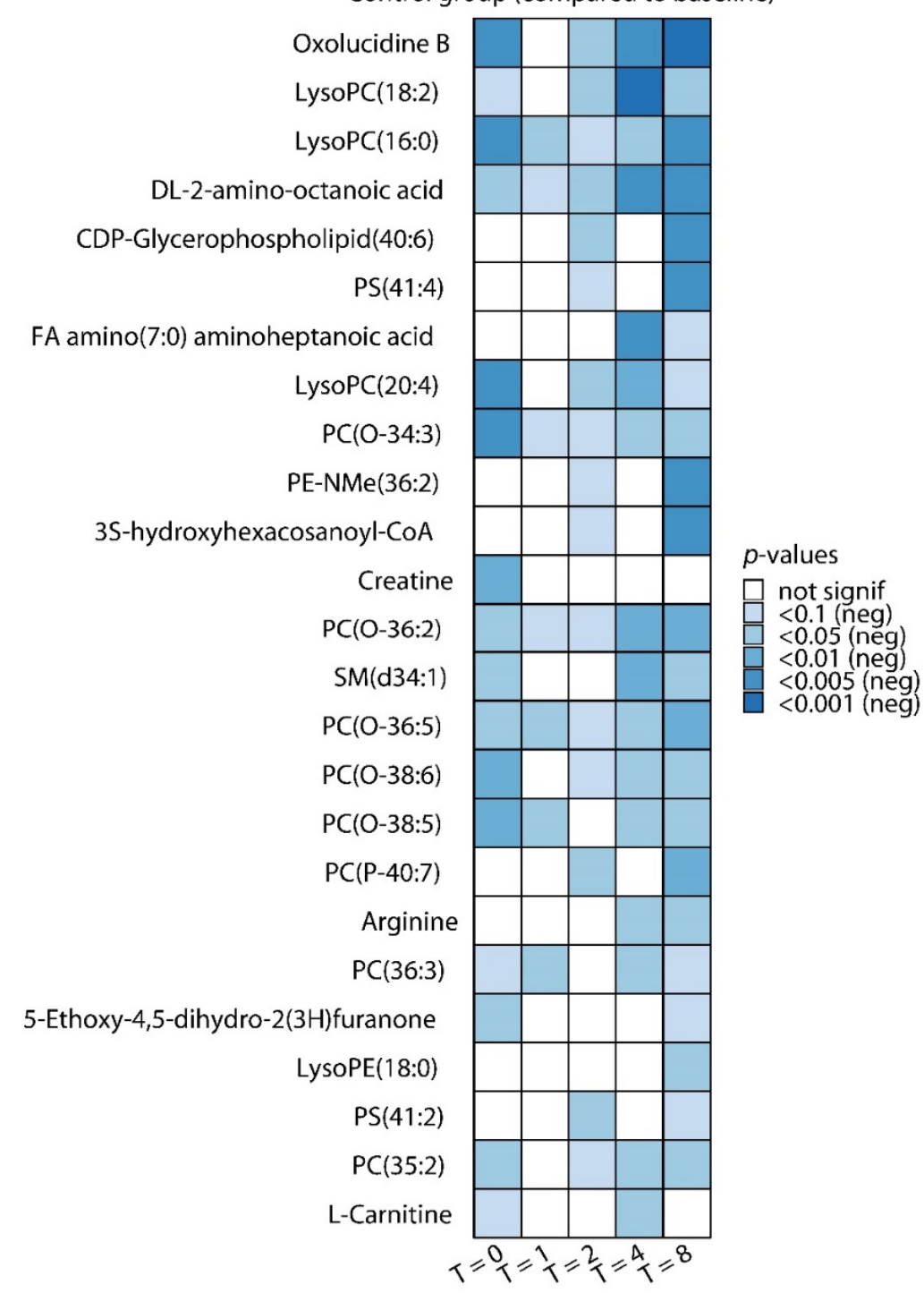

Figure 2. Top differentially regulated metabolites during endotoxemia. Top 25 most significantly (according to $p$-value) regulated metabolites at T $=0$ (just before LPS administration), 1, 2, 4, and 8 $\mathrm{h}$ post-LPS administration compared with baseline (one hour before LPS administration) in the control group. Blue color indicates a decrease compared with baseline (this top 25 only comprises decreased metabolites). $p$-values were calculated using paired $t$-tests with multiple-testing correction (Benjamini-Hochberg false discovery rate [FDR]).

The relatively large number of decreased metabolites at $\mathrm{T}=0$ is likely a result of dilution, as all subjects were pre-hydrated with $1.5 \mathrm{~L}$ of saline/glucose solution between baseline and $\mathrm{T}=0$ as part of our standard experimental endotoxemia protocol [8]. Because PCA and OPLS-DA models showed the most pronounced separation at $\mathrm{T}=4 \mathrm{~h}$, we focused on that timepoint, and all 44 significantly altered metabolites (10 increased, 34 decreased) at $\mathrm{T}=4 \mathrm{~h}$ are listed in Table 1. 
Table 1. Significantly altered metabolites $4 \mathrm{~h}$ after LPS administration in the control group.

\begin{tabular}{|c|c|c|c|}
\hline Metabolite & Class & $\begin{array}{c}\text { Fold Change } \\
\text { Versus Baseline }{ }^{1}\end{array}$ & $\begin{array}{c}\text { FDR Adjusted } \\
p \text {-Value }{ }^{2}\end{array}$ \\
\hline \multicolumn{4}{|l|}{ Increased } \\
\hline 5,8-Dihydro-6-(4-methyl-3-pentenyl)-1,2,3,4-tetrathiocin & Prenol lipids & +1.25 & 0.044 \\
\hline 6-Phosphonoglucono-D-lactone & Monosaccharides & +3.31 & 0.051 \\
\hline Deoxycytidine & Nucleosides, nucleotides, and derivatives & +24.77 & 0.061 \\
\hline Artonin K & Flavonoids & +1.43 & 0.065 \\
\hline Hexane-6-keto-1,3,4,6-tetracarboxylate & Unknown & +3.91 & 0.065 \\
\hline Cis-(homo)2aconitate & Metabolism of cofactors and vitamins & +1.25 & 0.071 \\
\hline Deoxyuridine triphosphate & Nucleosides, nucleotides, and derivatives & +4.46 & 0.076 \\
\hline Imidazoleacetic acid riboside & Nucleosides, nucleotides, and derivatives & +1.31 & 0.076 \\
\hline Lactic acid & Hydroxy acids and derivatives & +2.03 & 0.091 \\
\hline Gnidicin & Unknown & +6.87 & 0.096 \\
\hline \multicolumn{4}{|l|}{ Decreased } \\
\hline LysoPC(18:2) & Glycerophospholipids & -2.22 & 0.0008 \\
\hline DL-2-amino-octanoic acid & Amino acids and derivatives & -1.51 & 0.002 \\
\hline Oxolucidine B & Unknown & -2.27 & 0.005 \\
\hline FA amino(7:0) aminoheptanoic acid & Fatty acyls & -1.59 & 0.005 \\
\hline LysoPC(20:4) & Glycerophospholipids & -2.85 & 0.007 \\
\hline $\mathrm{PC}(\mathrm{O}-36: 2)$ & Glycerophospholipids & -1.26 & 0.007 \\
\hline $\mathrm{SM}(\mathrm{d} 34: 1)$ & Sphingolipids & -1.21 & 0.009 \\
\hline Arginine & Amino acids and derivatives & -1.40 & 0.013 \\
\hline $\mathrm{PC}(36: 3)$ & Glycerophospholipids & -1.29 & 0.015 \\
\hline PC(O-36:5) & Glycerophospholipids & -1.24 & 0.019 \\
\hline $\mathrm{PC}(\mathrm{O}-34: 3)$ & Glycerophospholipids & -1.28 & 0.021 \\
\hline L-Carnitine & Alkylamines & -1.32 & 0.022 \\
\hline Succinic acid semialdehyde & Fatty acids and conjugates & -1.47 & 0.022 \\
\hline PC(O-38:5) & Glycerophospholipids & -1.20 & 0.023 \\
\hline $\operatorname{PS}(21: 0)$ & Glycerophospholipids & -3.43 & 0.024 \\
\hline L-Acetylcarnitine & Fatty acid esters & -1.67 & 0.026 \\
\hline $\mathrm{PC}(\mathrm{O}-38: 6)$ & Glycerophospholipids & -1.21 & 0.027 \\
\hline Glutamine & Amino acids and derivatives & -1.45 & 0.028 \\
\hline $\mathrm{PC}(36: 5)$ & Glycerophospholipids & -1.65 & 0.035 \\
\hline PC(36:4) & Glycerophospholipids & -1.20 & 0.035 \\
\hline LysoPC(16:0) & Glycerophospholipids & -2.20 & 0.038 \\
\hline PC(P-40:6) & Glycerophospholipids & -1.19 & 0.045 \\
\hline PC(35:2) & Organic phosphoric acids and derivatives & -1.25 & 0.045 \\
\hline Narciclasine & Unknown & -1.86 & 0.045 \\
\hline $\mathrm{PC}(40: 6)$ & Glycerophospholipids & -1.69 & 0.045 \\
\hline Lenticin & Unknown & -1.35 & 0.065 \\
\hline $\mathrm{PC}(36: 2)$ & Glycerophospholipids & -1.35 & 0.066 \\
\hline PC(34:3) & Glycerophospholipids & -1.23 & 0.071 \\
\hline $\mathrm{PC}(38: 4)$ & Glycerophospholipids & -1.20 & 0.071 \\
\hline $\operatorname{PE}(39: 1)$ & Glycerophospholipids & -1.33 & 0.071 \\
\hline PC(O-34:2) & Glycerophospholipids & -1.30 & 0.071 \\
\hline $\mathrm{SM}(\mathrm{d} 34: 2)$ & Sphingolipids & -1.20 & 0.078 \\
\hline PC(38:5) & Glycerophospholipids & -1.17 & 0.087 \\
\hline TG(41:0) & Glycerolipids & -1.20 & 0.091 \\
\hline
\end{tabular}

${ }^{1}$ Baseline represents one hour before LPS administration. ${ }^{2} p$-values were calculated using paired $t$-tests with multiple-testing correction (Benjamini-Hochberg false discovery rate [FDR]).

Three of the increased metabolites belonged to the class of nucleosides, nucleotides, and derivatives. The majority of decreased metabolites were comprised of glycerophospholipids. Metabolite Set Enrichment Analysis (MSEA) [9] revealed that the most significantly affected pathways included glutamate metabolism, oxidation of various fatty acids, pyrimidine metabolism, the urea cycle, and the Warburg effect (Figure S1 in the Supplementary Material). 
2.2. Differences in Plasma Metabolites in Trained and Untrained Individuals

We performed PCA and constructed OPLS-DA models for the control versus trained group at each timepoint. PCA plots did not show clear separation (Figure 3a).

a
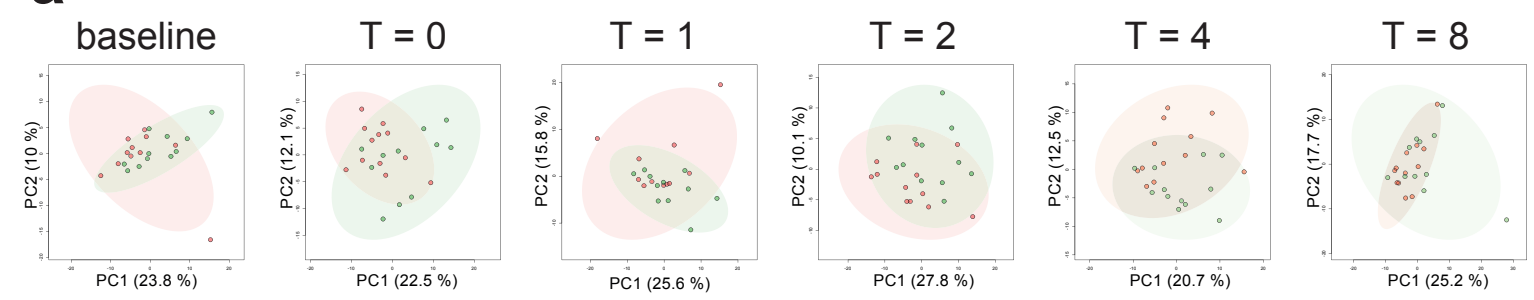

b

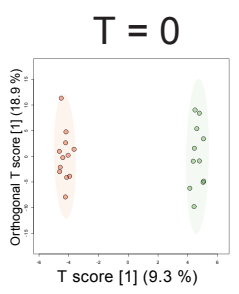

Figure 3. The plasma metabolome in trained and untrained subjects. (a) Principal component analysis (PCA) of the plasma metabolome at baseline (one hour before LPS administration, $\mathrm{T}=0$ (just before LPS administration), and 1, 2, 4, and $8 \mathrm{~h}$ post-LPS administration in the control (red dots) and trained (green dots) group. The shaded areas indicate the $95 \%$ confidence intervals. (b) Orthogonal partial least squares-discriminant analysis (OPLS-DA) of the plasma metabolome at $\mathrm{T}=0$ in the control (red dots) and trained (green dots) group. The shaded areas indicate the $95 \%$ confidence intervals.

Permutation tests revealed that the model constructed for the comparison between both groups at $\mathrm{T}=0 \mathrm{~h}$ (when the trained individuals had been practicing the breathing exercises for $30 \mathrm{~min}$ but LPS had not yet been administered) showed significant separation $\left(1+3\right.$ components, $R^{2} X_{\text {cum }}: 0.439$, $\mathrm{R}^{2} \mathrm{Y}_{\text {cum: }}: 0.995, \mathrm{Q}^{2}$ cum: 0.649; permutation tests: $\mathrm{R}^{2} \mathrm{Y}: p=0.03, \mathrm{Q}^{2}: p=0.001$, score plot depicted in Figure 3b), whereas models constructed at other timepoints did not. Differential analyses revealed a total of 19 significantly altered metabolites between trained and untrained groups across all timepoints (Figure 4). 


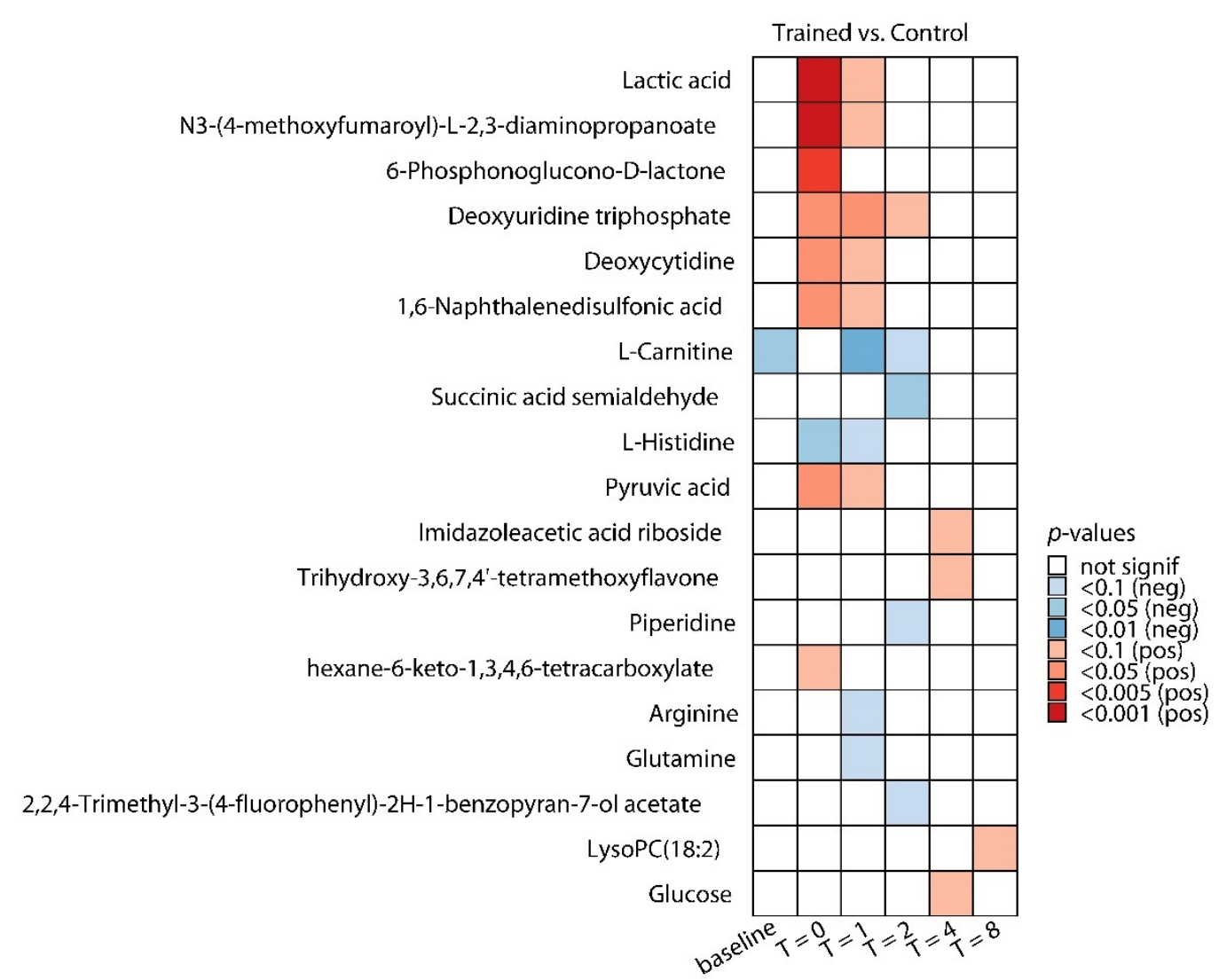

Figure 4. Differentially regulated metabolites in the trained group. Significantly regulated metabolites at baseline (one hour before LPS administration), $\mathrm{T}=0$ (immediately prior to LPS administration), and $1,2,4$, and $8 \mathrm{~h}$ following LPS administration in the training group compared with the control group. Blue color indicates a decrease compared with the control group, red color indicates an increase compared with the control group. $p$-values were calculated using unpaired $t$-tests with multiple-testing correction (Benjamini-Hochberg false discovery rate [FDR]).

At baseline (after the trained subjects had completed the training program but before any endotoxemia-related procedures or breathing exercises were performed), L-carnitine was lower in the trained group. Nevertheless, the vast majority of significant between-group changes were observed at timepoints between $\mathrm{T}=0$ and $\mathrm{T}=2 \mathrm{~h}$, during which the trained group practiced the breathing exercises acquired during their training program. A list of all metabolites across all timepoints in both groups is provided in Tables S3 and S4 in the Supplementary Material. Based on the OPLS-DA model that showed significant separation between both groups at $\mathrm{T}=0$, we focused on that particular timepoint, and all 9 significantly altered metabolites ( 8 up, 1 down) at $\mathrm{T}=0$ are listed in Table 2. 
Table 2. Significantly altered metabolites between the trained and control group at $\mathrm{T}=0$ (30 min after initiation of the breathing exercises and prior to LPS administration).

\begin{tabular}{|c|c|c|c|}
\hline Metabolite & Class & $\begin{array}{c}\text { Fold-Change } \\
\text { (Trained/Control) }\end{array}$ & $\begin{array}{c}\text { FDR-Adjusted } \\
p \text {-Value }{ }^{1}\end{array}$ \\
\hline \multicolumn{4}{|l|}{ Increased } \\
\hline N3-(4-methoxyfumaroyl)-L-2,3-diaminopropanoate & Unknown & +3.15 & 0.0007 \\
\hline Lactic acid & Carbohydrate metabolism & +3.00 & 0.0007 \\
\hline 6-Phosphonoglucono-D-lactone & Lactones & +3.19 & 0.005 \\
\hline Deoxyuridine triphosphate & Nucleosides, nucleotides, and derivatives & +4.91 & 0.006 \\
\hline Deoxycytidine & Nucleosides, nucleotides, and derivatives & +9.15 & 0.007 \\
\hline 1,6-Naphthalenedisulfonic acid & Unknown & +2.19 & 0.007 \\
\hline Pyruvic acid & Alcohols and polyols & +2.07 & 0.035 \\
\hline Hexane-6-keto-1,3,4,6-tetracarboxylate & Unknown & +3.52 & 0.083 \\
\hline \multicolumn{4}{|l|}{ Decreased } \\
\hline L-Histidine & Amino acids and derivatives & -2.72 & 0.026 \\
\hline
\end{tabular}

${ }^{1} p$-values were calculated using unpaired $t$-tests with multiple-testing correction (Benjamini-Hochberg false discovery rate [FDR]).

Lactic acid (lactate) was one of the most significantly increased metabolites in the trained group. We previously measured this metabolite at the bedside using a point-of-care blood analyzer (i-STAT) [1], which provided us with an opportunity to validate the liquid chromatography-mass spectrometry (LC-MS) analysis for this metabolite. Lactate concentration measurements were virtually identical between the two methods in both groups (Figure S2A,B in the Supplementary Material). Furthermore, there was excellent correlation between both methods, with only a single outlier sample observed ( $\mathrm{r}$ values of 0.90 and 0.97 with and without the outlier sample in the analysis, respectively, Figure S2C in the Supplementary Material). Two other metabolites that were increased (deoxyuridine triphosphate and deoxycytidine) belong to the nucleosides, nucleotides, and derivates class, while 6-phosphonoglucono-D-lactone is an intermediate in the pentose phosphate pathway, and pyruvic acid (pyruvate) is a well-known metabolite of the alcohols and polyols class. The only decreased metabolite was L-histidine, an amino acid used in the biosynthesis of proteins. MSEA enrichment analysis revealed that the most significantly affected pathways included ammonia recycling, pyruvate metabolism, the Warburg effect, pyrimidine metabolism, gluconeogenesis, glutamate metabolism, and amino sugar metabolism (Figure 5). 


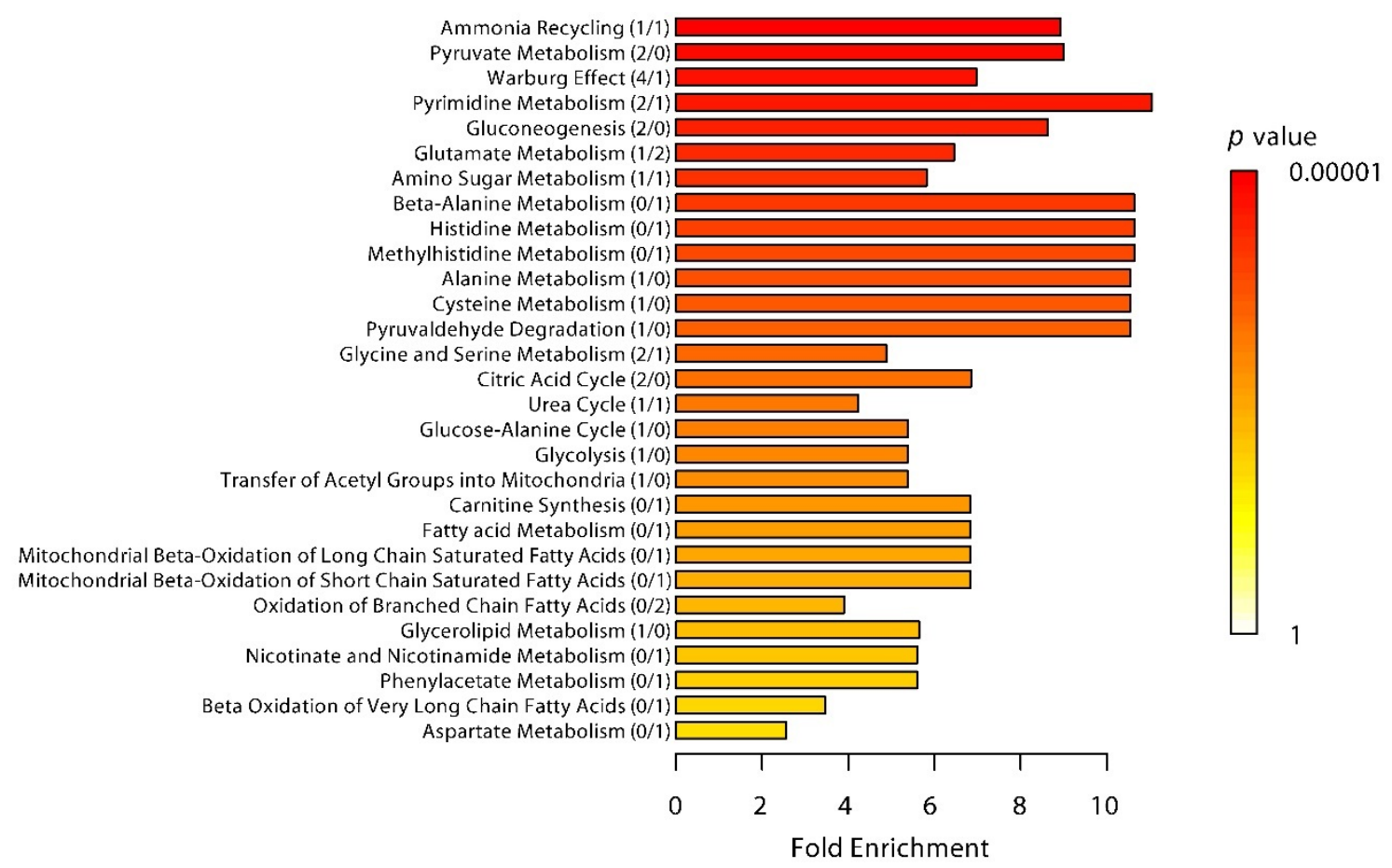

Figure 5. Enriched pathways in the trained group. Significantly enriched pathways at $\mathrm{T}=0$ in the trained group compared with the control group. Threshold for significance was set at a Benjamini-Hochberg false discovery rate [FDR]-adjusted $p$-value of less than 0.1 . Number of significantly increased/decreased metabolites within each pathway are indicated in parentheses.

\subsection{Relationship between Differentially Regulated Metabolites and Inflammatory Response Mediators}

We evaluated whether in trained individuals practicing the breathing exercises, plasma concentrations of the significantly altered metabolites listed in Table 2 at early timepoints correlated with in vivo cytokine responses, which were profoundly modulated in these subjects (i.e., strongly increased plasma levels of the anti-inflammatory cytokine IL-10 and attenuated concentrations of pro-inflammatory cytokines tumor necrosis factor (TNF) $\alpha$, IL-6, and IL-8, see Table S2 in the Supplementary Material and Reference [1]). At $\mathrm{T}=0$, only one significant correlation was found, between L-histidine and IL-8 $(r=0.62, p=0.048)$. At $\mathrm{T}=1$, significant correlations between several metabolites and plasma levels of IL-10, which were enhanced by three-fold in trained subjects compared with the control group (Table S2 in the Supplementary Material and Reference [1]), were found. These include 1,6-naphthalenedisulfonic acid ( $\mathrm{r}=0.76, p=0.02)$, deoxyuridine triphosphate $(\mathrm{r}=0.78, p=0.03)$, and deoxycytidine $(\mathrm{r}=0.74, p=0.03)$. Interestingly, also lactate and pyruvate levels at $\mathrm{T}=1$ correlated with the AUC IL-10 response $(r=0.60, p=0.04$ and $r=0.66, p=0.03$, respectively, Figure $6 a, b)$. 

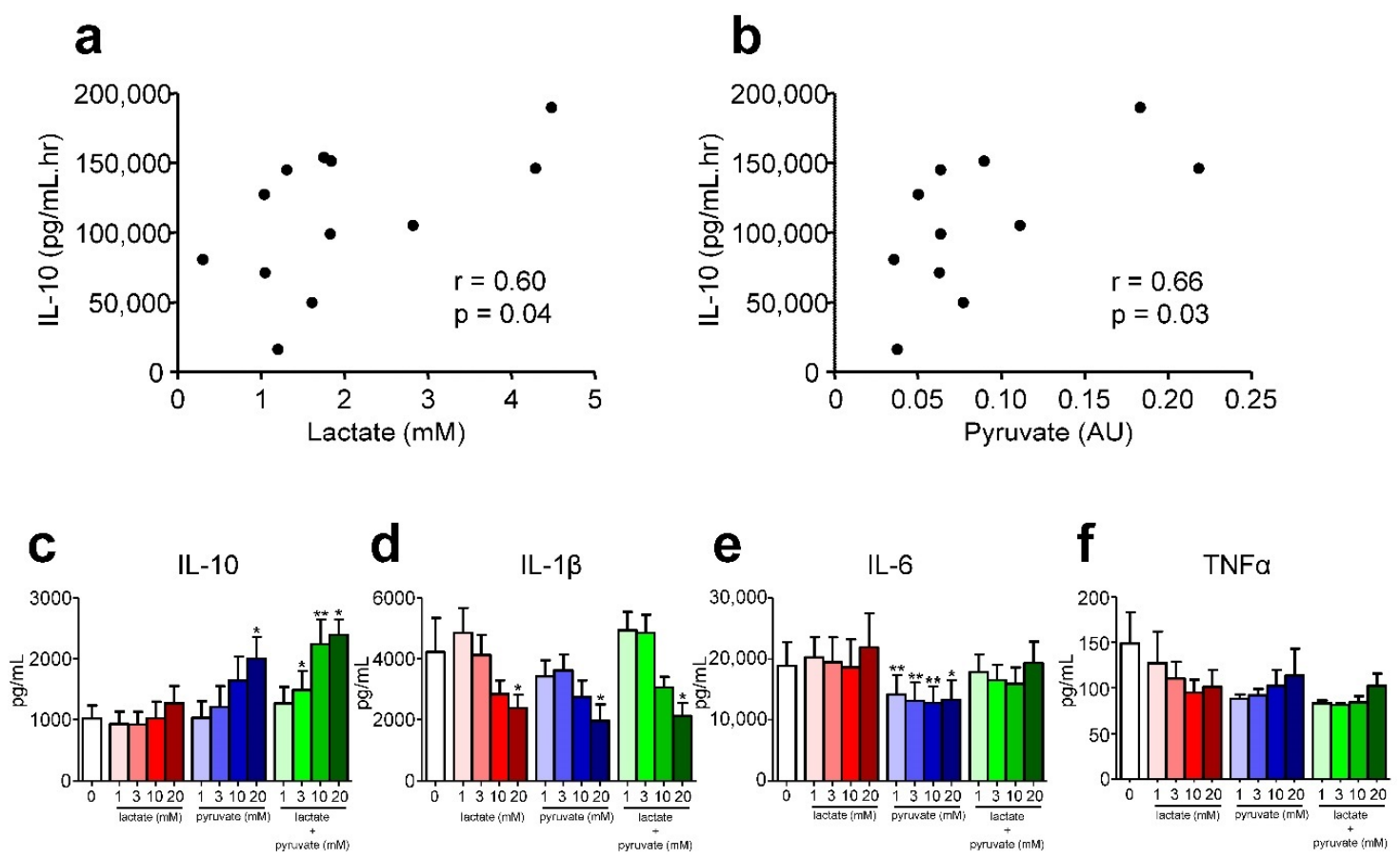

Figure 6. Role of lactate and pyruvate in interleukin (IL)-10 induction. (a-b) Relationship between plasma lactate (a) and pyruvate (b) levels at $\mathrm{T}=1$ and the plasma IL-10 response (expressed as area under the time-concentration curve (AUC)). (c-f) IL-10, IL-1 $\beta$, IL-6 and tumor necrosis factor (TNF) $\alpha$ production by LPS-stimulated peripheral blood mononuclear cells (PBMCs) incubated with medium alone (white bar) or different concentrations of lactate (red bars), pyruvate (blue bars), or a combination of the two (green bars). Spearman correlation was used to calculate $\mathrm{r}$ and $p$-values in panels a and b. Data in panels $c-f$ are depicted as mean \pm standard error of the mean (SEM) of 6 (panels c-e) or 3 (panel f) donors. ${ }^{*} p<0.05,{ }^{* *} p<0.01$ versus medium control (paired $t$-tests).

Furthermore, these two metabolites were highly intercorrelated $(\mathrm{r}=0.94, p<0.0001)$, but were not related to the profoundly increased epinephrine levels observed in trained subjects practicing the breathing exercises at any of the measured timepoints ( $p$-values $>0.15)$, which were previously shown to be an important driver of enhanced IL-10 production [1]. Because both lactate and pyruvate have been implicated to exert anti-inflammatory effects $[10,11]$, we set out to validate the observed associations using in vitro experiments with peripheral blood mononuclear cells (PBMCs) obtained from healthy individuals. High concentrations of pyruvate, but not lactate, enhanced LPS-induced IL-10 production, and the combination of both metabolites resulted in an even more pronounced and statistically robust increase (Figure 6c). Furthermore, pyruvate attenuated production of the pro-inflammatory cytokines IL-1 $\beta$ and IL-6 (Figure $6 \mathrm{~d}, \mathrm{e}$ ), whereas high concentrations of lactate as well as the combination of both metabolites mitigated IL-1 $\beta$ production (Figure $6 \mathrm{~d}$ ). LPS-induced $\mathrm{TNF} \alpha$ production was rather low, it was detectable in cell culture supernatants of only three out of the six healthy donors (data of these subjects are shown in Figure 6f). This is likely due to the fact that the $48 \mathrm{~h}$ incubation time is too prolonged to reliably measure TNF $\alpha$ responses, as levels in supernatants were previously shown to decline as early as $6 \mathrm{~h}$ following LPS stimulation [12]. Nevertheless, lactate, pyruvate, and the two metabolites combined also tended to attenuate LPS-induced TNF $\alpha$ production, although significance was not reached, probably due to the low remaining sample size.

\section{Discussion}

In the present work, we demonstrated that the systemic inflammatory response induced by LPS in healthy volunteers significantly alters the plasma metabolome, with the most profound changes taking place $4 \mathrm{~h}$ following LPS administration. Endotoxemia mainly led to an increase of several nucleosides, 
nucleotides, and derivatives, and a decrease in many glycerophospholipids. Before LPS administration, trained subjects practicing the learned breathing exercises exhibited higher levels of lactate and pyruvate compared with a control group who did not practice any exercise, and concentrations of these metabolites correlated with the profoundly enhanced levels of the anti-inflammatory cytokine IL-10 observed in trained individuals following LPS administration. We subsequently validated these findings in vitro, by showing that co-incubation with lactate and pyruvate enhances LPS-induced IL-10 release and attenuates pro-inflammatory cytokine production by primary human leukocytes.

Two of the most profoundly enhanced metabolites after the LPS challenge were deoxyuridine triphosphate and especially, deoxycytidine, both involved in the pyrimidine metabolism pathway, which was enriched following LPS administration. Increased plasma deoxycytidine levels result from DNA degradation [13]. We have previously shown that plasma levels of both nuclear and mitochondrial DNA show a transient increase during endotoxemia, reaching their maximum levels $3 \mathrm{~h}$ after LPS administration, after which they gradually return to baseline in the following hours [14]. Hence, it might be speculated that the breakdown of plasma DNA is one of the main drivers of the increased deoxycytidine concentrations observed $4 \mathrm{~h}$ after LPS administration. Many metabolites belonging to the class of glycerophospholipids were distinctly decreased following administration of LPS. Related to this, various pathways related to lipid metabolism were enriched, which is in accordance with previous findings obtained in healthy volunteers undergoing experimental endotoxemia [15]. Glycerophospholipids are the main components of cell membranes and function as precursors to signaling molecules involved in many cellular and physiological processes [16]. The majority of the decreased metabolites belonging to this class consisted of phosphatidylcholines. Previous work from our group has shown that plasma levels of secretory phospholipase A2 (sPLA $)_{2}$, the principal catalysts of glycerophospholipid hydrolysis, greatly increase during endotoxemia [17], which may be an explanation for the reduced plasma glycerophospholipid concentrations. The Warburg effect is another pathway of interest that was enriched during systemic inflammation, with four significantly increased (6-phosphonoglucono-D-lactone, glucose, lactate, pyruvate) and one significantly decreased (L-glutamine) metabolite. This process entails the shift from oxidative phosphorylation (OXPHOS) as the primary energy source towards aerobic glycolysis and was recently shown to play a critical role in mounting (LPS-induced) immune responses, by, among other things, facilitating rapid production of inflammatory cytokines [18-20].

Following the training program, trained subjects exhibited lower plasma levels of L-carnitine compared with untrained controls. This relative depletion of carnitine from plasma may indicate an overall increment in Acyl-shuttling mechanism in the mitochondria by CPT1/CPT2 and might be related with lipid beta-oxidation intensification [21]. The most pronounced changes in the plasma metabolome of trained subjects compared with controls were, however, observed during the period in which the breathing exercises were carried out. This was not unexpected given the major changes in cardiorespiratory parameters, markers of autonomic nervous system activity, and inflammatory molecules and symptoms observed during this period, as reported elsewhere [1]. At $\mathrm{T}=0$, when the trained individuals had been practicing the breathing exercises for $30 \mathrm{~min}$ but just prior to LPS administration, the Warburg effect was one of the top enriched pathways in trained individuals compared with controls. Plasma levels of four metabolites (lactate, 6-phosphonoglucono-D-lactone, pyruvate, and isocitric acid) of this pathway were increased in the trained group, whereas concentrations of L-glutamine were lower. This finding may be counterintuitive, as the Warburg effect is mainly associated with pro-inflammatory effects, whereas trained subjects exhibited a distinct anti-inflammatory phenotype [1]. The fact that LPS, a strong inducer of the Warburg effect [20], had not yet been administered at this early timepoint, renders this finding biologically implausible as well. Finally, as alluded to before, the Warburg effect encompasses a shift from oxidative phosphorylation (and thus usage of the citric acid cycle) towards glycolysis, but the citric acid cycle pathway was also enhanced in the trained group. Therefore, we hypothesize that the observed effect is not due to a true Warburg effect (aerobic glycolysis), but rather driven by the profoundly increased lactate levels 
in this group as a result of classical anaerobic glycolysis in the muscles. The latter may have been caused by the combination of vigorous breathing (including repeated muscle tightening), intermittent hypoxia, and epinephrine-induced vasoconstriction in this group [1]. The breathing exercises could certainly be regarded as exercise, given that, similar to exercise, they resulted in profuse sweating and exhaustion. The fact that lactate and pyruvate levels were highly intercorrelated provides a strong indication of increased gluconeogenesis (i.e., the generation of glucose from non-carbohydrate substrates such as lactate, with pyruvate as the intermediate metabolite, which mainly takes place in the liver). Gluconeogenesis was also one of the top enhanced pathways in the trained group during practicing of the breathing exercises. Because epinephrine is a well-known strong inducer of gluconeogenesis [22], the profoundly increased plasma epinephrine levels observed shortly after initiation of the breathing exercises [1] likely play a pivotal role in this effect. Combining the findings of enhanced anaerobic glycolysis with increased gluconeogenesis, our data provide evidence of increased activation of the Cori cycle in trained subjects during practicing of the breathing exercises, in which lactate is produced by muscle cells and subsequently converted back, via pyruvate, into glucose in the liver [23]. However, it needs to be acknowledged that we can only infer activation of the Cori cycle, as we did not perform isotype labeling experiments.

Correlation analysis between differentially regulated metabolites and inflammatory cytokine levels in trained subjects revealed that both lactate and pyruvate were related to plasma concentrations of the key anti-inflammatory cytokine IL-10. Of note, production of this cytokine was profoundly accelerated and enhanced in trained subjects practicing the breathing exercises, and its plasma levels were strongly correlated to the subsequent attenuation of pro-inflammatory cytokine responses [1]. Our PBMC stimulation experiments demonstrated that pyruvate alone, but especially the combination of both pyruvate and lactate, exert a robust enhancing effect on LPS-induced IL-10 production. These results may substantiate the correlations found during endotoxemia in vivo. Furthermore, both lactate and pyruvate individually, as well as the combination of both metabolites, attenuate IL- $1 \beta$ production, and lactate mitigated the release of IL-6, another pro-inflammatory cytokine. Both lactate and pyruvate have been demonstrated previously to exert anti-inflammatory effects. For instance, very recent work demonstrates that addition of lactate to LPS-stimulated primary human monocytes causes a distinct metabolic shift by decreasing aerobic glycolysis and increasing oxidative phosphorylation, a metabolic state characteristic for anti-inflammatory responses [24]. In the same study, lactate was shown to decrease LPS-induced production of pro-inflammatory cytokines by human PBMCs [24]. The authors proposed that immunomodulatory effects of lactate may serve as a feedback signal to limit excessive inflammatory responses of highly glycolytic pro-inflammatory immune cells [24]. Pyruvate has been shown to decrease mRNA expression and protein levels of pro-inflammatory cytokines TNF $\alpha$ and IL-6 in LPS-stimulated canine PBMCs, whereas IL-10 expression and production was increased [25]. Furthermore, administration of pyruvate significantly lowered IL-6 and enhanced IL-10 plasma concentrations in LPS-treated rats, leading to prolonged survival, and incubation with ethyl pyruvate blocked activation of nuclear factor (NF)- $\mathrm{KB}$, a critical pro-inflammatory transcription factor, in LPS-stimulated murine macrophages [26]. These data strengthen the notion that also in the human in vivo situation, the observed increases in lactate and pyruvate at least partly account for the immunomodulatory effects observed in trained subjects.

Several limitations and aspects of the present work deserve attention. First, only male volunteers were included. In earlier work, we observed that the endotoxin-induced pro-inflammatory immune response is more pronounced in females than in males [27]. Furthermore, menstrual cycle-induced variation in hormone levels can also impact immune parameters [28,29], thereby further increasing variation. Therefore, inclusion of both sexes yields more inter-individual variation and would necessitate larger group sizes. Because endotoxemia studies are very labor-intensive and costly, the choice was made to only include males, which nevertheless limits this study's generalisability.

A second limitation, which applies to all plasma metabolomics studies, pertains to the uncertainty of the source of the measured metabolites. Virtually all identified metabolites can be produced by a 
wide variety of cells in many organs. Especially in immunological studies like this, combining plasma metabolomics with the determination of intracellular metabolites in immune cells (e.g., leukocytes) would represent a more powerful approach. Unfortunately, no samples were stored for this purpose.

Third, the current study does not allow us to deduce which (combination) of the three elements of the training program is responsible for the observed effects, and several of our ongoing studies are aimed at elucidating this question. Nevertheless, as alluded to in a previous section, the breathing exercises probably play a pivotal role.

Fourth, because metabolomic profiling was performed on samples stored for three years at $-80^{\circ} \mathrm{C}$, sample degradation could be an issue. Nevertheless, the high correlation between lactate measured during the experiments using a point-of-care analyzer and by LC-MS indicates that, at least for this metabolite, no significant degradation occurred during storage. Furthermore, because storage time was virtually identical across samples (all were collected within one month), the extent of degradation of certain metabolites would have been similar across all samples. Provided that metabolites did not completely degrade, this would therefore have little impact on the results.

Fifth, as the PBMCs stimulation experiments with lactate and pyruvate contained a mixture of different cell types (predominantly monocytes and lymphocytes), we cannot be certain about the cellular origin of the effects observed. However, it is well established that monocytes are the main cytokine producers in short-term whole blood LPS stimulation assays (which next to PBMCs also contain granulocytes) [30]. Furthermore, as discussed earlier on, anti-inflammatory effects of lactate and pyruvate have been reported in primary human monocytes [24] and murine macrophages [26], the latter of which show many similarities to monocytes. Therefore, it is likely that the effects of pyruvate and lactate we observed can predominantly be ascribed to monocytes.

Finally, and importantly, all training procedures described in this study were conducted in the presence of medical personnel. Because of profound physiological effects of the breathing exercises (e.g., acid-base shifts, intermittent hypoxia) and exposure to cold [1], potential health risks while practicing these elements of the training program should be considered.

In conclusion, the present study extends our previous findings regarding the effect of a training intervention consisting of cold exposure, breathing exercises, and meditation on the LPS-induced immune response in healthy volunteers. Practicing the breathing exercises acquired during the training program results in enhanced activity of the Cori cycle, and next to the previously established relationship between epinephrine and IL-10 induction [1], the current data indicate a role of lactate and pyruvate in the enhanced production of this key anti-inflammatory mediator and in the overall anti-inflammatory phenotype observed in trained subjects.

\section{Materials and Methods}

\subsection{Subjects and Experimental Design}

Metabolomic profiling was performed in plasma samples obtained in a previously published parallel randomized controlled study registered at ClinicalTrials.gov as NCT01835457 [1]. The study protocol is described in detail elsewhere [1]. Briefly, after approval from the local ethics committee of the Radboud University Medical Center (CMO 2012/455), 24 healthy nonsmoking male volunteers with a median age of 22 years (range 19-27) provided written informed consent and were included in the study. All study procedures were in accordance with the Declaration of Helsinki, including current revisions, and Good Clinical Practice guidelines. Subjects were screened before the study and had a normal physical examination, electrocardiography, and routine laboratory values. Exclusion criteria were: febrile illness during the 2 weeks before the endotoxemia experiment, taking any prescription medication, history of spontaneous vagal collapse, practicing or experience with any kind of meditation, or participation in a previous trial where LPS was administered. The subjects were randomly allocated to the trained group $(n=12)$ or the control group $(n=12)$ by the opening of sealed envelopes prepared by unblinded staff not involved in the study. The trained group underwent a 10 day 
training program provided by Dutch individual Wim Hof, which consisted of three main elements: meditation, exposure to cold, and breathing exercises (see Reference [1] for a detailed description). After completion of the training, subjects of both groups $(n=24)$ underwent experimental human endotoxemia at our intensive care research unit, consisting of administration of an intravenous bolus of $2 \mathrm{ng} / \mathrm{kg}$ of US Standard Reference Escherichia coli endotoxin (E. coli O:113 (LPS), Clinical Center Reference Endotoxin; National Institutes of Health, Bethesda, MD, USA). As part of our standard endotoxemia protocol [31,32], the subjects received $1.5 \mathrm{~L} 0.9 \% \mathrm{NaCl}$ over one hour, starting one hour before endotoxin infusion (pre-hydration), followed by $150 \mathrm{~mL} / \mathrm{h}$ until $6 \mathrm{~h}$ after endotoxin infusion and $75 \mathrm{~mL} / \mathrm{h}$ until the end of the experiment. The experimental human endotoxemia protocol is provided in detail elsewhere [1,32]. The control group did not undergo any training procedures and also underwent experimental human endotoxemia. Subjects in the trained group started practicing the breathing exercises acquired during the training program $30 \mathrm{~min}$ before LPS administration until two-and-a-half $\mathrm{h}$ afterwards. The control group did not practice any exercise throughout the endotoxemia experiment. As described in detail elsewhere [1], the breathing exercises consisted of two exercises. In brief, the first exercise comprised cycles of vigorous hyperventilation (approximately 30 breaths) followed by breath holding for several minutes at the discretion of the subject. The second exercise was similar, but at the end of the hyperventilation period, breath was only held for $10 \mathrm{~s}$ during which all body muscles were tightened.

\subsection{Sample Preparation for LC-MS Analysis}

Ethylenediaminetetraacetic acid (EDTA) anti-coagulated blood was obtained one hour before LPS administration (baseline), and at $\mathrm{T}=0, \mathrm{~T}=1, \mathrm{~T}=2, \mathrm{~T}=4$, and $\mathrm{T}=8 \mathrm{~h}$. Blood was immediately centrifuged at $2000 \mathrm{~g}$ for $10 \mathrm{~min}$ at $4{ }^{\circ} \mathrm{C}$, after which plasma was stored at $-80^{\circ} \mathrm{C}$ until analysis. Sample extraction was performed as described previously [7], with some modifications. Briefly, $50 \mu \mathrm{L}$ of plasma was mixed with $450 \mu \mathrm{L}$ of $90 \%(v / v)$ methanol containing internal standards and incubated for $15 \mathrm{~min}$ at $37^{\circ} \mathrm{C}$ with $1150 \mathrm{rpm}$. Precipitated proteins were separated from the extract by centrifugation for $12 \mathrm{~min}$ at $15,000 \mathrm{rpm}$, after which supernatants were stored at $-80^{\circ} \mathrm{C}$ until further analysis.

\subsection{LC-MS Analysis}

Modified reversed-phase chromatography in combination with high resolution mass spectrometry (HRMS) was employed in this study. Samples were analyzed on an Agilent 1290 ultra high performance liquid chromatography (UPLC) system (Agilent) with a Discovery HS F5-3 column $(15 \mathrm{~cm} \times 2.1 \mathrm{~mm}$, $3 \mu \mathrm{m}$, Supelco, Sigma Aldrich) coupled to a high-resolution 6540 quadrupole time-of-flight mass spectrometry (QTOF/MS) detector (Agilent) operated in both positive and negative electrospray ionization (ESI) mode in a detection range of 50 to $1700 \mathrm{~m} / z$ at $2 \mathrm{GHz}$ in extended dynamic range. The liquid chromatography (LC) solvent consisted of $95 \% 10 \mathrm{mM}$ ammonium formate with $0.1 \%$ formic acid and 5\% acetonitrile (A), and 95\% acetonitrile with 5\% $10 \mathrm{mM}$ ammonium formate with $0.1 \%$ formic acid (B). A multi-step gradient was used, with $5 \% \mathrm{~B}$ from $0-0.1 \mathrm{~min}$ to $35 \% \mathrm{~B}$ at $1.5 \mathrm{~min}$, to $95 \% \mathrm{~B}$ at $2.05 \mathrm{~min}$ which was kept constant until $3.2 \mathrm{~min}$, to $5 \% \mathrm{~B}$ at $3.21 \mathrm{~min}$ and washing until $4.3 \mathrm{~min}$ with $5 \% \mathrm{~B}$. The flow rate was kept constant at $700 \mu \mathrm{L} / \mathrm{min}$ from 0 to $2.2 \mathrm{~min}$, and increased up to $900 \mu \mathrm{L} / \mathrm{min}$ from 2.2 to $2.5 \mathrm{~min}$, after which flow rate was kept constant until $3.2 \mathrm{~min}$. The flow rate was decreased from 900 to $800 \mu \mathrm{L} / \mathrm{min}$ from 3.2 to $3.1 \mathrm{~min}$ and kept constant until $3.7 \mathrm{~min}$, when the flow rate was changed to $700 \mu \mathrm{L} / \mathrm{min}$. The run time was $4.3 \mathrm{~min}, 1 \mu \mathrm{l}$ of sample was injected and the column heated to $40{ }^{\circ} \mathrm{C}$. The DualAJS ESI source was set to the following parameters: Gas temperature $200{ }^{\circ} \mathrm{C}$, drying gas $8 \mathrm{~L} / \mathrm{min}$, nebulizer 35 pounds-force per square inch gauge (psig), sheath gas temp: $350{ }^{\circ} \mathrm{C}$, sheath gas flow $11 \mathrm{~L} / \mathrm{min}$, capillary voltage (VCap) $3500 \mathrm{~V}$ and nozzle voltage of $0 \mathrm{~V}$. Online calibration of the instrument was performed throughout the data acquisition using Agilent ES-TOF Reference Mass Solution Kit. 


\subsection{Cytokine and Lactate Determinations}

Plasma concentrations of the cytokines TNF $\alpha$, IL-6, IL-8, and IL-10 at various timepoints during the endotoxemia experiment were determined by Luminex assay (Milliplex, Millipore), as described previously [1]. The area under the cytokine plasma concentration-time curves (AUC) was used as an integral measure of the subjects' in vivo cytokine responses. Furthermore, we validated the LC-MS analysis for lactate by comparing LC-MS data with lactate concentrations measured by a point-of-care blood analyzer (i-STAT, Abbot), as described previously [1].

\subsection{PBMC Stimulation Experiments}

After approval from the local ethics committee of the Radboud university medical center (CMO 2010/10), EDTA anticoagulated blood was obtained from 6 healthy donors. Isolation of PBMCs was performed by differential centrifugation over Ficoll-Paque PLUS (GE Healthcare Biosciences) in SepMate tubes (STEMCELL technologies). PBMCs were washed thrice with phosphate buffered saline (PBS), counted, and $5 \times 10^{5} \mathrm{PBMCs} /$ well were seeded in 96-well round-bottom plates in Roswell Park Memorial Institute (RPMI) 1640 culture medium (Dutch Modification, Invitrogen) supplemented with $50 \mu \mathrm{g} / \mathrm{mL}$ gentamycin (Thermo Fisher Scientific) and $2 \mathrm{mM}$ Glutamax (Invitrogen). Cells were incubated with RPMI (control), or 1, 3, 10, or $20 \mathrm{mM}$ of sodium lactate (provided by the Department of Pharmacy, Radboud University Medical Center, Nijmegen, The Netherlands), pyruvate (Invitrogen), or a combination of sodium lactate and pyruvate for $1 \mathrm{~h}$ at $37^{\circ} \mathrm{C}$ and $5 \% \mathrm{CO}_{2}$. Subsequently, RPMI or $10 \mathrm{ng} / \mathrm{mL}$ E. coli-derived LPS (serotype O55:B5, Sigma Aldrich) was added and PBMCs were incubated for $48 \mathrm{~h}$ at $37^{\circ} \mathrm{C}$ and $5 \% \mathrm{CO}_{2}$. Concentrations of IL-10, IL-1 $\beta$, IL-6, and TNF $\alpha$ in the cell culture supernatants were measured by enzyme-linked immunosorbent assay (ELISA, R\&D systems).

\subsection{Raw Data Processing and Statistical Analyses}

Chromatograms were generated by the LC-MS instrument in .d format. Raw data were converted into mzXML and chromatogram peaks were extracted using XCMS v1.42.0 [33], which was optimized using the IPO R package [34] with the following settings: peakwidth $=c(10,70), \mathrm{ppm}=20$, snthresh $=10$, mzdiff $=0.0034$, prefilter $=c(3,100)$, noise $=100$, gapInit $=0.8448$, gapExtend $=2.0544, \mathrm{bw}=5$, mzwid $=0.015, \operatorname{minfrac}=0.5, \max =50$. All further analyses were performed in $\mathrm{R}$ programming language [35], Metaboanalyst 4.0 [9], and GraphPad Prism version 5.0 (GraphPad Software). IDEOM software (http://mzmatch.sourceforge.net/ideom.php) [36] was used to eliminate noise and for putative peak annotation by exact mass within \pm 10 ppm against the Metabolomic Discoveries in the house metabolite library [37] in negative and positive ESI mode, respectively. Retention time prediction was applied to aid metabolite annotation. 972 peaks were detected. Non-annotated metabolites and potential peptides were removed $(n=748)$, leaving 224 metabolites in the dataset. Furthermore, 6 outlier samples were identified using ROBPCA by defining the sample distances within the orthogonal to the projection plane [38]. This left a total of 138 samples to be analyzed. Principal component analysis (PCA) and orthogonal partial least squares-discriminant analysis (OPLS-DA) were performed to visualize the metabolic alterations between samples after log-transformations followed by mean-centering and dividing by the standard deviation of each variable. OPLS-DA models were validated by permutation tests (1000 permutations) [39]. Differential analyses were performed using paired (within-group comparisons) and unpaired (between-group comparisons) $t$-tests with multiple-testing correction using Benjamini-Hochberg false discovery rate (FDR) [40]. Analogous to previous work [15,41], a FDR-adjusted $p$-value of 0.1 was set as a threshold for statistical significance in the differential analyses. Targeted analyses (permutation tests, Spearman correlation between metabolites and cytokines, and paired $t$-tests for the PBMC stimulation data) were performed without applying the FDR correction, and the threshold for significance was set at $p<0.05$ for these data. We restricted correlation analyses to metabolite levels at early timepoints $(T=0$ and $T=1)$, because these can still 
relevantly affect the immunological response, which is orchestrated in the first hour and peaks 1.5 to 2 $\mathrm{h}$ after LPS administration [1].

The metabolomics dataset is available at the National Institutes of Health (NIH) Common Fund's National Metabolomics Data Repository (NMDR) website, the Metabolomics Workbench, https://www.metabolomicsworkbench.org, where it has been assigned Project ID PR00083. The data can be accessed directly via it's Project DOI: 10.21228/M8C671. Metabolomics Workbench is supported by NIH grant U2C-DK119886.

Supplementary Materials: The following are available online at http://www.mdpi.com/2218-1989/10/4/148/s1: Figure S1: Enriched pathways after LPS administration, Figure S2: Comparison of plasma lactate concentrations measured by LC-MS and a point-of-care analyzer. Table S1: Subject characteristics, Table S2: Baseline, peak, and area under curve (AUC) plasma cytokine responses in control and trained groups, Table S3: Changes in plasma metabolite levels during endotoxemia in the control group. Table S4: Changes in plasma metabolite levels during endotoxemia in the trained group.

Author Contributions: Conceptualization, J.R., R.S., P.P., and M.K.; formal analysis, J.Z., R.t.H., I.B., D.S., and M.K.; investigation, J.Z., I.B., D.S., and M.K.; writing-original draft preparation, J.Z., R.t.H., I.B., D.S., and M.K.; writing-review and editing, J.M.W., N.S., R.S., M.G.N., D.J., and P.P.; supervision, J.M.W., N.S., R.S., M.G.N., D.J., P.P., and M.K.; funding acquisition, P.P. and M.K. All authors have read and agreed to the published version of the manuscript.

Funding: The plasma samples used for analysis in the present work were collected in a study performed by J.Z., P.P., and M.K., which was supported by a Serendipity Grant from the Dutch Arthritis Society (ReumaNederland, https://reumanederland.nl/), grant no. 12-1-101.

Acknowledgments: We greatly appreciate the help of Manuela Hische and Michael Heiser for their support with the analysis, and Judith Lenk and Monique Thiele for the technical assistance.

Conflicts of Interest: During the conduct of this study, I.B., D.S., J.W., and N.S. were employed by Metabolomic Discoveries GmbH, Potsdam, Germany. This company was acquired by Metabolon in September 2017. The remaining authors declare no conflict of interest. The funders had no role in the design of the study; in the collection, analyses, or interpretation of data; in the writing of the manuscript, or in the decision to publish the results.

\section{References}

1. Kox, M.; van Eijk, L.T.; Zwaag, J.; van den Wildenberg, J.; Sweep, F.C.; van der Hoeven, J.G.; Pickkers, P. Voluntary activation of the sympathetic nervous system and attenuation of the innate immune response in humans. Proc. Natl. Acad. Sci. USA 2014, 111, 7379-7384. [CrossRef] [PubMed]

2. Bravo, E.L. Metabolic factors and the sympathetic nervous system. Am. J. Hypertens. 1989, 2, 339S-344S. [CrossRef] [PubMed]

3. Stienstra, R.; Netea-Maier, R.T.; Riksen, N.P.; Joosten, L.A.B.; Netea, M.G. Specific and Complex Reprogramming of Cellular Metabolism in Myeloid Cells during Innate Immune Responses. Cell Metab. 2017, 26, 142-156. [CrossRef] [PubMed]

4. O'Neill, L.A.; Kishton, R.J.; Rathmell, J. A guide to immunometabolism for immunologists. Nat. Rev. Immunol. 2016, 16, 553-565. [CrossRef]

5. Schwertner, H.A.; Kong, S.B. Determination of modafinil in plasma and urine by reversed phase high-performance liquid-chromatography. J. Pharm. Biomed. Anal. 2005, 37, 475-479. [CrossRef]

6. Mirsaeidi, M.; Banoei, M.M.; Nienow, C.K.; Abassi, T.; Hakim, A.; Schraufnagel, D.; Winston, B.W.; Sweiss, N.; Baughman, R.; Garcia, J.G.; et al. Plasma metabolomic profile in fibrosing pulmonary sarcoidosis. Sarcoidosis Vasc. Diffus. Lung Dis. Off. J. WASOG World Assoc. Sarcoidosis Granul. Disord. 2016, 33, $29-38$.

7. Van de Beek, M.C.; Dijkstra, I.M.; van Lenthe, H.; Ofman, R.; Goldhaber-Pasillas, D.; Schauer, N.; Schackmann, M.; Engelen-Lee, J.Y.; Vaz, F.M.; Kulik, W.; et al. C26:0-Carnitine Is a New Biomarker for X-Linked Adrenoleukodystrophy in Mice and Man. PLoS ONE 2016, 11, e0154597. [CrossRef]

8. Van Eijk, L.T.; Pickkers, P.; Smits, P.; Bouw, M.P.; van der Hoeven, J.G. Severe vagal response after endotoxin administration in humans. Intensive Care Med. 2004, 30, 2279-2281. [CrossRef]

9. Xia, J.; Wishart, D.S. Web-based inference of biological patterns, functions and pathways from metabolomic data using MetaboAnalyst. Nat. Protoc. 2011, 6, 743-760. [CrossRef] 
10. Nolt, B.; Tu, F.; Wang, X.; Ha, T.; Winter, R.; Williams, D.L.; Li, C. Lactate and Immunosuppression in Sepsis. Shock 2018, 49, 120-125. [CrossRef]

11. Fink, M.P. Ethyl pyruvate: A novel anti-inflammatory agent. J. Intern. Med. 2007, 261, 349-362. [CrossRef] [PubMed]

12. DeForge, L.E.; Remick, D.G. Kinetics of TNF, IL-6, and IL-8 gene expression in LPS-stimulated human whole blood. Biochem. Biophys. Res. Commun. 1991, 174, 18-24. [CrossRef]

13. Cohen, J.D.; Strock, D.J.; Teik, J.E.; Katz, T.B.; Marcel, P.D. Deoxycytidine in human plasma: Potential for protecting leukemic cells during chemotherapy. Cancer Lett. 1997, 116, 167-175. [CrossRef]

14. Timmermans, K.; Kox, M.; Scheffer, G.J.; Pickkers, P. Plasma Nuclear and Mitochondrial DNA Levels, and Markers of Inflammation, Shock, and Organ Damage in Patients with Septic Shock. Shock 2016, 45, 607-612. [CrossRef] [PubMed]

15. Kamisoglu, K.; Sleight, K.E.; Calvano, S.E.; Coyle, S.M.; Corbett, S.A.; Androulakis, I.P. Temporal metabolic profiling of plasma during endotoxemia in humans. Shock 2013, 40, 519-526. [CrossRef]

16. Quehenberger, O.; Dennis, E.A. The human plasma lipidome. N. Engl. J. Med. 2011, 365, 1812-1823. [CrossRef]

17. Dinkla, S.; van Eijk, L.T.; Fuchs, B.; Schiller, J.; Joosten, I.; Brock, R.; Pickkers, P.; Bosman, G.J. Inflammation-associated changes in lipid composition and the organization of the erythrocyte membrane. BBA Clin. 2016, 5, 186-192. [CrossRef]

18. Zheng, J.I.E. Energy metabolism of cancer: Glycolysis versus oxidative phosphorylation (Review). Oncol. Lett. 2012, 4, 1151-1157. [CrossRef]

19. Cheng, S.C.; Joosten, L.A.; Netea, M.G. The interplay between central metabolism and innate immune responses. Cytokine Growth Factor Rev. 2014, 25, 707-713. [CrossRef]

20. Tannahill, G.M.; Curtis, A.M.; Adamik, J.; Palsson-McDermott, E.M.; McGettrick, A.F.; Goel, G.; Frezza, C.; Bernard, N.J.; Kelly, B.; Foley, N.H.; et al. Succinate is an inflammatory signal that induces IL-1beta through HIF-1alpha. Nature 2013, 496, 238-242. [CrossRef]

21. Longo, N.; Frigeni, M.; Pasquali, M. Carnitine transport and fatty acid oxidation. Biochim. Biophys. Acta 2016, 1863, 2422-2435. [CrossRef] [PubMed]

22. Sacca, L.; Vigorito, C.; Cicala, M.; Corso, G.; Sherwin, R.S. Role of gluconeogenesis in epinephrine-stimulated hepatic glucose production in humans. Am. J. Physiol. 1983, 245, E294-E302. [CrossRef] [PubMed]

23. Hoffer, L.J. Cori cycle contribution to plasma glucose appearance in man. JPEN J. Parenter. Enter. Nutr. 1990, 14, 646-648. [CrossRef] [PubMed]

24. Ratter, J.M.; Rooijackers, H.M.M.; Hooiveld, G.J.; Hijmans, A.G.M.; de Galan, B.E.; Tack, C.J.; Stienstra, R. In vitro and in vivo Effects of Lactate on Metabolism and Cytokine Production of Human Primary PBMCs and Monocytes. Front. Immunol. 2018, 9, 2564. [CrossRef]

25. Yu, D.H.; Noh, D.H.; Song, R.H.; Park, J. Ethyl pyruvate downregulates tumor necrosis factor alpha and interleukin (IL)-6 and upregulates IL-10 in lipopolysaccharide-stimulated canine peripheral blood mononuclear cells. J. Vet. Med. Sci. 2010, 72, 1379-1381. [CrossRef]

26. Venkataraman, R.; Kellum, J.A.; Song, M.; Fink, M.P. Resuscitation with Ringer's ethyl pyruvate solution prolongs survival and modulates plasma cytokine and nitrite/nitrate concentrations in a rat model of lipopolysaccharide-induced shock. Shock 2002, 18, 507-512. [CrossRef]

27. Van Eijk, L.T.; Dorresteijn, M.J.; Smits, P.; van der Hoeven, J.G.; Netea, M.G.; Pickkers, P. Gender differences in the innate immune response and vascular reactivity following the administration of endotoxin to human volunteers. Crit. Care Med. 2007, 35, 1464-1469. [CrossRef]

28. O’Brien, S.M.; Fitzgerald, P.; Scully, P.; Landers, A.; Scott, L.V.; Dinan, T.G. Impact of gender and menstrual cycle phase on plasma cytokine concentrations. Neuroimmunomodulation 2007, 14, 84-90. [CrossRef]

29. Gornicsar, K.; Mozes, T.; Grosz, A.; Biro, E.; Ladi, S.; Clayton, P. TNFalpha Variation During the Menstrual Cycle and Thereafter: A New Explanation for Gender-Based Disparities in ICU Admission Rates, Trauma Outcomes, and General Mortality. Shock 2017, 47, 416-421. [CrossRef]

30. Segre, E.; Fullerton, J.N. Stimulated Whole Blood Cytokine Release as a Biomarker of Immunosuppression in the Critically Ill: The Need for a Standardized Methodology. Shock 2016, 45, 490-494. [CrossRef]

31. Dorresteijn, M.J.; van Eijk, L.T.; Netea, M.G.; Smits, P.; van der Hoeven, J.G.; Pickkers, P. Iso-osmolar prehydration shifts the cytokine response towards a more anti-inflammatory balance in human endotoxemia. J. Endotoxin Res. 2005, 11, 287-293. [CrossRef] [PubMed] 
32. Van Lier, D.; Geven, C.; Leijte, G.P.; Pickkers, P. Experimental human endotoxemia as a model of systemic inflammation. Biochimie 2018, 159, 99-106. [CrossRef] [PubMed]

33. Smith, C.A.; Want, E.J.; O’Maille, G.; Abagyan, R.; Siuzdak, G. XCMS: Processing mass spectrometry data for metabolite profiling using nonlinear peak alignment, matching, and identification. Anal. Chem. 2006, 78, 779-787. [CrossRef] [PubMed]

34. Libiseller, G.; Dvorzak, M.; Kleb, U.; Gander, E.; Eisenberg, T.; Madeo, F.; Neumann, S.; Trausinger, G.; Sinner, F.; Pieber, T.; et al. IPO: A tool for automated optimization of XCMS parameters. BMC Bioinform. 2015, 16, 118. [CrossRef]

35. R Core Team. R: A Language and Environment for Statistical Computing. Available online: https: //www.r-project.org (accessed on 16 June 2016).

36. Creek, D.J.; Jankevics, A.; Burgess, K.E.; Breitling, R.; Barrett, M.P. IDEOM: An Excel interface for analysis of LC-MS-based metabolomics data. Bioinformatics 2012, 28, 1048-1049. [CrossRef]

37. Brand, B.; Hadlich, F.; Brandt, B.; Schauer, N.; Graunke, K.L.; Langbein, J.; Repsilber, D.; Ponsuksili, S.; Schwerin, M. Temperament type specific metabolite profiles of the prefrontal cortex and serum in cattle. PLoS ONE 2015, 10, e0125044. [CrossRef]

38. Stoessel, D.; Schulte, C.; Teixeira Dos Santos, M.C.; Scheller, D.; Rebollo-Mesa, I.; Deuschle, C.; Walther, D.; Schauer, N.; Berg, D.; Nogueira da Costa, A.; et al. Promising Metabolite Profiles in the Plasma and CSF of Early Clinical Parkinson's Disease. Front. Aging Neurosci. 2018, 10, 51. [CrossRef]

39. Bijlsma, S.; Bobeldijk, I.; Verheij, E.R.; Ramaker, R.; Kochhar, S.; Macdonald, I.A.; van Ommen, B.; Smilde, A.K. Large-scale human metabolomics studies: A strategy for data (pre-) processing and validation. Anal. Chem. 2006, 78, 567-574. [CrossRef]

40. Benjamini, Y.; Hochberg, Y. Controlling the False Discovery Rate: A Practical and Powerful Approach to Multiple Testing. J. R. Stat. Soc. 1995, 57, 289-300. [CrossRef]

41. Kamisoglu, K.; Calvano, S.E.; Coyle, S.M.; Corbett, S.A.; Androulakis, I.P. Integrated transcriptional and metabolic profiling in human endotoxemia. Shock 2014, 42, 499-508. [CrossRef]

(C) 2020 by the authors. Licensee MDPI, Basel, Switzerland. This article is an open access article distributed under the terms and conditions of the Creative Commons Attribution (CC BY) license (http://creativecommons.org/licenses/by/4.0/). 